\title{
Morphological characteristics of X-ray thorax images of COVID-19 patients using the Bradley thresholding segmentation
}

\author{
Retno Supriyanti ${ }^{1}$, Muhammad Alqaaf ${ }^{2}$, Yogi Ramadhani ${ }^{3}$, Haris B. Widodo ${ }^{4}$ \\ ${ }^{1,2,3}$ Electrical Engineering Department, Jenderal Soedirman University, Purwokerto, Indonesia \\ ${ }^{4}$ Medical Faculty, Jenderal Sooedirman University, Purwokerto, Indonesia
}

\begin{tabular}{l} 
Article Info \\
\hline Article history: \\
Received May 6, 2021 \\
Revised Sep 9, 2021 \\
Accepted Sep 16, 2021 \\
\hline Keywords: \\
Bradley thresholding \\
Computer aided diagnosis \\
COVID-19 \\
Thorax \\
X-ray images
\end{tabular}

\begin{abstract}
The coronavirus disease 2019 (COVID-19) pandemic has made test screening much needed. Currently, the most commonly used is the swab type. Although in fact, there is also a screening method with chest radiology. The purpose of this study is to develop a COVID-19 early detection system based on X-ray images of the patient's thorax in the form of a computer-aided diagnosis. This case is based on the fact that X-ray modalities are available in several health care centers in Indonesia, compared to other modalities such as computed tomography (CT) scan or magnetic resonance imaging (MRI). In this paper, we emphasize the X-ray thorax image segmentation process to explore the morphological information of the thorax. We use the Bradley thresholding segmentation method. The results obtained are promising to be further developed with a performance percentage of $73.33 \%$ for the thorax for COVID-19 patients and 54\% for the thorax for normal patients.
\end{abstract}

This is an open access article under the CC BY-SA license.

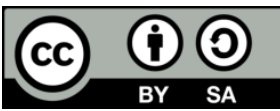

\section{Corresponding Author:}

Retno Supriyanti

Department of Electrical Engineering, Jenderal Soedirman University

Kampus Blater, St. Mayjend Sungkono KM 5 Blater Purbalingga, Indonesia

Email: retno_supriyanti@unsoed.ac.id

\section{INTRODUCTION}

The World Health Organization (WHO) officially declared the coronavirus (COVID-19) a pandemic in 2020. In general, the coronavirus causes mild or moderate symptoms, such as fever and cough, and most can recover within a few weeks. However, for some people at high risk (the elderly and people with chronic health problems, such as heart disease, high blood pressure, or diabetes), the coronavirus can cause serious health problems. Most of the victims come from that risk group. So it is essential to understand how to reduce these risks. According to the WHO report in March 2021, the number of Indonesia's population confirmed to be affected by COVID-19 is 1,511,712 persons; this number is significant in a disease epidemic [1].

In humans, the thorax has a vital role in exchanging oxygen $(\mathrm{O} 2)$ and carbon monoxide $(\mathrm{CO} 2)$. The COVID-19 virus spreads through droplet droplets from an infected person, then enters the body of the nearby person through the mouth, nose, and eyes. The virus then got on track respiratory tract and mucous membranes at the back of the throat, attached to a receptor in the lung cells, and then develops there. The initial symptoms that result from infection with the COVID-19 virus are starting pain in the back of the throat. Then, the COVID-19 virus quickly propagates into the base of the thorax to enter thorax. Process this damages the lung tissue so that the tissue becomes filled with fluid, pus, and dead cells [2]. Conventional testing is to detect whether someone is infected by one of the ways for the COVID-19 virus is to carry out testing polymerase chain reaction (PCR) swab test. PCR swab examination using mucus samples taken from 
the nose or throat. The two areas were chosen because they were the places where the virus reproduced itself. This examination is considered more accurate because the coronavirus will stick to the part in the nose or throat as it enters the body. The result of this PCR swab examination will show its existence SARS-CoV-2 virus in a person's body. To carry out a diagnosis more in-depth and knowing the condition of the sufferer's thorax, is done taking images of his thorax through the X-ray method [3]. On the X-ray results of the thorax of people with COVID-19, the fluid contained inside the lungs will look like white spots. Fluid sufferers of COVID-19 are similar to cases of pneumonia and difficulty syndrome acute respiratory distress syndrome. This fluid buildup is on in the thorax, causing the sufferer to have difficulty breathing, even the worst condition that can cause death [4]. Along with the increasing number of cases infected with COVID-19, handling must be done immediately to increase casualties because of this pandemic. Speed, accuracy, and accuracy in screening for COVID-19 are critical.

Many studies discuss the detection of COVID-19, including the following. Ragnesola et al. [5] discussed the need for sustainable COVID-19 data for monitoring and overcoming this pandemic. He used the lateral flow assay (LFA) testing platform, using seropositivity on 63 plasma convaselen donor (CP) samples to determine IgG and IgM. CP donors show mixed results. Gill et al. [6] aims to create a complete profile of the leukocytes of COVID-19 patients and compare these profiles with healthy non-COVID-19 patients. The method used is to make ribonucleic acid sequences (RNAseq) expressed differently for later identification. The results obtained were that COVID-19 patients who were critical on the first day provided a unique leukocyte profile. Goshen-Lago et al. [7] researched cancer patients receiving COVID-19 serological treatment to estimate their immunity pattern to SARS-CoV2. Alshazly et al. [8] in his research, adopted the use of network architecture in a series of experiments with two sets of CT image data, namely the SARS-CoV-2 and COVID-19-CT scans. His research shows promising results in achieving accuracy, precision, specificity, and sensitivity values. Siwiak et al. [9] researched by developing a meta populationtype SIR transmission model to determine the simulation of the spread of the COVID-19 pandemic in the New York area. Tiwari and Jain [10] developed an early detection system to differentiate pneumonia from COVID-19 based on the CapsNet network system. Cho et al. [11] developed a vaccine system using recombinant baculoviral, DNA-based, to protect the coronavirus syndrome in the Middle East. Leger et al. [12] developed a system to determine the severity of COVID-19 based on CT scan images of the lungs. Wang et al. [13] applied the Convolutional Neural Network in the detection of COVID19 based on X-ray images. Jaffe et al. [14] discussed clinical and ethical challenges in liver transplantation and proposed guidance for end-stage patient management during the COVID-19 pandemic. Connell et al. [15] provided recommendations regarding the management of head and neck cancer during the COVID-19 pandemic. Dhamad and Rhida [16] conducted a review of molecular and serological methods for the detection of COVID-19. Keihanian and Bigdelu [17] conducted a comprehensive review of cardiovascular disorders and their complications from exposure to COVID-19. The INRIA group of COVID-19 [18] evaluates the initial needs of the COVID-19 service unit at the hospital, such as the need for a bed in the ICU unit not to be displaced even though the number is booming due to the COVID-19 pandemic. As with our previous research [19]-[23] which has always focused on using digital image processing technology for health services in rural areas, we will develop a detection of COVID-19 based on image processing techniques in this research. In the early stages, we will segment the thorax based on the results of X-ray radiological images using the Bradley segmentation method. We used a standard thorax model from Ercleve [24] as a reference to determine thoracic abnormalities in COVID-19 sufferers. Several other research that use thorax imagery in identifying COVID-19 are the use of the internet of things (IoT) [25], adaptive stratification [26], social approachment [27], blood cell ratio [28], thoraxic surgery [29], tomography images [30], otolaryngology surgery [31], handheld optical system [32], SOM-LWL method [33], therapeutic effects [34], upper respiratory tract [35].

\section{PROPOSED METHOD}

Bradley thresholding is a thresholding method formed from the development of the adaptive thresholding method by Derek Bradley. This method works by comparing the pixels to the nearest pixel, keeping hard contrast lines, and ignoring soft gradient changes. If the current pixel value t percent is lower than average, it will be set to black; otherwise, it will be set to white. The advantage of this method is that only one processing of the image is required, and it is more robust against changing illumination of the image and is simple and easy to apply. However, there is one major problem with this method in that the binarization results depend on the sequence in which the pixels are scanned [36]. Referring to the weakness of this method, in its use in this research, we carry out a series of pre-processing so that the image to be segmented can match the Bradley segmentation algorithm. In this research, we use the Bradley segmentation method because of the advantages when applied to images with uneven illumination. This case follows the 
X-ray Thorax image condition in patients with COVID-19 that we used in this research. However, there are some modifications in using this Bradley segmentation method, which we describe in section 3.

\section{METHOD}

\subsection{Data acquisition}

In this research, we used two types of data in the form of X-ray images of the thorax from COVID-19 patients and normal patients. The image data of COVID-19 patients comes from the Italian Society of Medical nd Interventional radiology. Meanwhile, normal patients data comes from the pages www.kaggle.com, nihcc.app.box.com, and radiopaedia.org. Figure 1 shows some examples of input data used in this research.
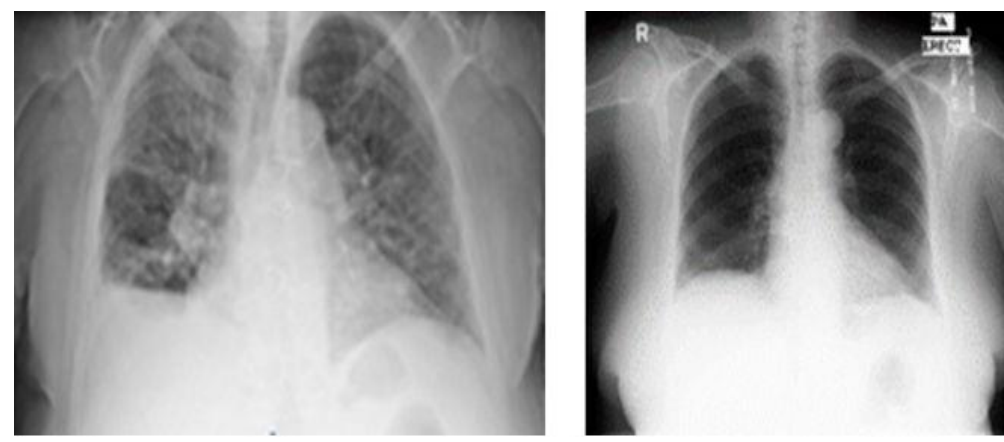

Figure 1. The examples of input image

\subsection{Pre-processing}

The input data used in this experiment do not have the appropriate qualifications with the system we are developing, so preprocessing is necessary. The aim is to improve image quality by removing existing noise, increasing contrast, brightness, sharpening the intensity of the object's edges, and eliminating the blurry effect. Some of the methods used in the preprocessing process are cropping to select specific parts that will be needed and resizing to equalize the image size so that it is the same size.

- Image resize

Resize has the goal of adjusting the size of the image so that the size is uniform between images. The data obtained has an image size with different pixels, so there is a need for an equalization of the size so that when doing detection, the results are more accurate, and the system can run as desired. For example, Figure 2 compares the size of the original image and the resized image. Figure 2 is an example of resizing an image to a size of 500x500 pixels, Figure 2(a). The original image has a size of 1249x1243 pixels, and Figure 2(b) the resized image has a size of 500x500 pixels.

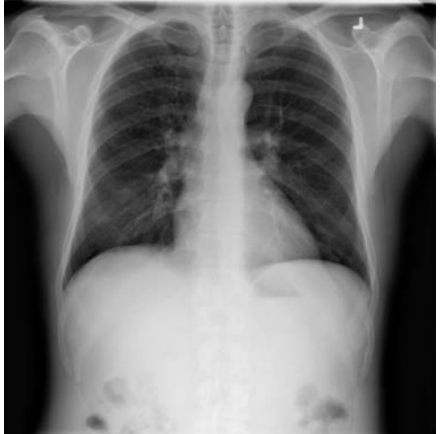

(a)

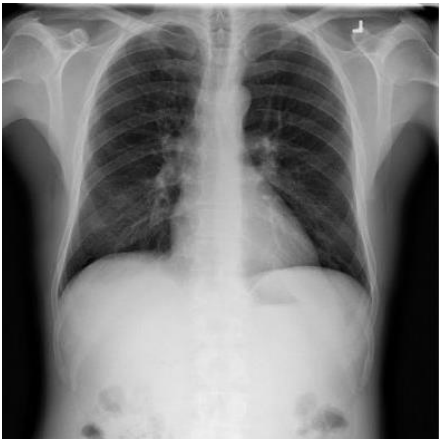

(b)

Figure 2. Rezising an image; (a) original image and (b) resize image

\section{- Cropping}

Cropping aims to reduce the size of an image by cutting the image at predetermined coordinates on an area in the image. In this research, only the thorax areas are needed. In addition, part of the thorax will be 
removed so that the system's work becomes more focused on the thorax, and the detection becomes more accurate because the area of coverage is specific. The cropping area is based on the anatomy of the thorax X-ray image, where the X-ray results consist of three organs, namely the thorax, heart, and stomach [24]. In this experiment, the cropping method used is the polygon method. This method aims to select the part of the lung to be studied against other organs that are not included in the research. Figure 3 localizes only the thorax area that will be analyzed using Bradley thresholding in this research. Figure 3 shows the cropping process we did in order to get only the thorax area. Figure 3(a) original image and Figure 3(b) cropped image.

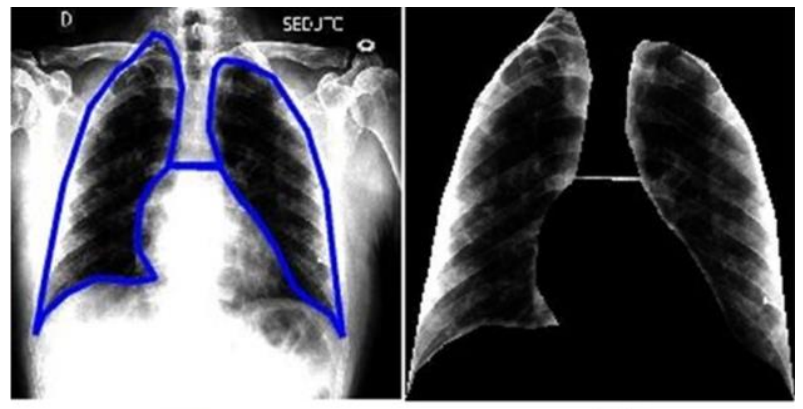

(a)

(b)

Figure 3. An example of cropping process; (a) original image and (b) cropped image

\subsection{Bradley thresholding}

Segmentation of the thorax and white spots was done using the Bradley thresholding method. In the Bradley thresholding method, the pixels in the image are compared with the pixels around it. The comparison will produce two different colors in the image, namely black and white. The Bradley thresholding method calculates the average window s x s pixels centered around each pixel. This method is a better average for comparison considering neighboring pixels on all sides. Calculation of the average is done in linear time using an integral image. Then this method calculates the integral image in the first path through the input image. Furthermore, the average pixel s x s is calculated using the integral image for each pixel in constant time, and then comparisons are made. If the comparison results show that the current pixel value $t$ percent is smaller than this average, it is set to black. Otherwise, it is set to white [36]. Using this method as a segmentation method is that only one process is required on the required image, and this method is more robust against changes in illumination in images and is simple and easy to apply. In this experiment, 95 image samples will be tested, consisting of 45 samples of thorax X-rays of the COVID-19 sufferers and 50 samples of thorax X-rays of normal patients.

\section{RESULTS AND DISCUSSIONS}

The reason for choosing a resize to the size of 500x500 pixels is that when using the actual pixel size, the percentage of test results produced is not much different from the image that has been resized by 500x500 pixels. The comparison of the test results is as shown in Table 1. Referring to Table 1, comparing the percentage of test results between the image with the actual pixel size and the image with the resized pixel size is not much different. So from that, to get faster and more efficient results, testing using 500x500 pixel resize is chosen because if we use an image with pixel size, it takes quite a bit of time to process it, especially when loading the input image into the system it takes a very long time for an image with that size. The next step is to crop the resized input image as described in the subsection above. Table 2 shows an example of the cropping we have done.

Table 1. Comparison of process between original size thorax X-ray image and resized image

\begin{tabular}{cccccc}
\hline \multirow{2}{*}{ No. } & \multirow{2}{*}{ Image } & \multicolumn{2}{c}{ Original Image } & \multicolumn{2}{c}{ Image Resize (500x500 pixels) } \\
& & Percentage of Test Results & Time Processing & Percentage of Test Results & Percentage of Test Results \\
\hline 1. & Image_Covid1 & 56,4043 & 00.55 & 57,6321 & 00.31 \\
2. & Image_Covid2 & 56,7299 & 00.62 & 55,1183 & 00.34 \\
3. & Image_Covid3 & 65,3266 & 00.70 & 65,0124 & 00.33 \\
4 & Image_Normal1 & 46,1738 & 00.55 & 46,8432 & 00.35 \\
5. & Image_Normal2 & 47,9675 & 00.68 & 44,0977 & 00.30 \\
6 & Image_Normal3 & 49,618 & 00.60 & 48,9084 & 00.37 \\
\hline
\end{tabular}


Referring to Table 2, We can be seen in the original image that there are still parts of the body from the results of image data retrieval, so cropping is carried out so that the detection process is only carried out on the desired object, namely the thorax. The form of cropping varies in each lung image data because every human being has a different thorax shape. The next step is segmentation which is at the core of this experiment. As explained above, we use Bradley segmentation to localize the thorax area to be explored morphological information. Table 3 shows some examples of thorax segmentation results using the Bradley segmentation method.

Table 2. Cropping results

\begin{tabular}{ccc}
\hline No & Image & Cropping area \\
\hline 2. Image_Covid1 & Image_Covid2 \\
3. Image_Covid3 & Image_Normal1 \\
5. Image_Normal2 & Image_Normal3
\end{tabular}

The resulting image from the Bradley thresholding segmentation is, as shown in Figure 3, then labeled information that can be explored, especially in the white patches on the thorax. The white patches objects in the segmented thorax X-ray image are labeled. The goal is to get the number of white patches objects, the area of each white patches, and the total area of the white patches. Figure 4 shows an example of the result of the labeling process in the white spot in the thorax.

Figure 4 is the labeling result of the image sample of thorax with COVID-19, where the system has detected 163 white patches with a total area of 24084 pixels. The labeling results, as shown in Figure 4, are obtained through several stages. The first stage determines the coordinate points, and the number of white spots on all detected white spots; then, parameters such as centroid, area, perimeter, and rectangle coordinates are sought from these coordinate points. Furthermore, these parameters can be used to describe labeling with a red rectangle. A rectangle can be created from a bounding box by knowing the coordinates $\mathrm{x} 1, \mathrm{x} 2$, and $\mathrm{y} 2$. We use (1) to calculate the percentage of the white patches as in Figure 4.

$$
\text { white patches percentage }=\frac{\text { white patches area }}{\text { thorax area }} \times 100 \%
$$

The amount of data used in this research is 95 sample images, which are divided into 45 images of COVID-19 thorax and 50 images of normal thorax. This research experiment hypothesized that the thorax 
X-ray images of COVID-19 sufferers had a smaller thorax area than the normal. In addition, it also has a broader area of white patches, a more comprehensive number of white patches, and a higher percentage of white patches than that found in normal thorax X-ray images. After testing, there are several images whose results are not following the hypothesis that has been mentioned above. This case can be caused by the quality of the image data itself. Image quality has various parameters, such as brightness, sharpness, and image resolution. In this experiment, if the system has performed the preprocessing operation well, but the results obtained are not good, then the possibility of the problem can come from the image data itself. The possibility of this problem could be in the form of a quality parameter that the image itself is not good at so that it influences the segmentation process also affects the research results because it does not produce optimal segmentation. In addition, the image quality can be influenced by the quality factor of the X-ray machine and the quality of the film used as the media for X-ray photos. Some examples of research results on COVID-19 thorax X-rays and normal thorax X-rays that do not match the hypothesis are shown in Figure 5.

Table 3. Segmentation results

\begin{tabular}{lcl} 
No & Image & Cropping Result Thorax segmentation White patches segmentation \\
\hline 1. Image_Covid1 & Image_Covid2 \\
3. Image_Covid3 & Image_Normal1 \\
5. Image_Normal2 & Image_Normal3
\end{tabular}

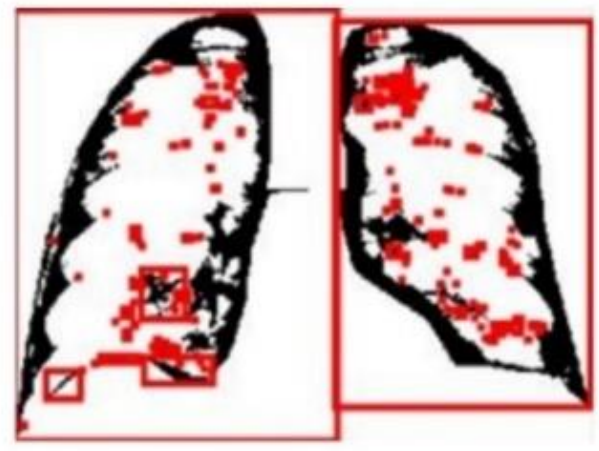

Figure 4. An example of labeling on the thorax COVID-19

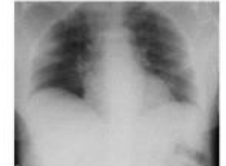

Failed covid19

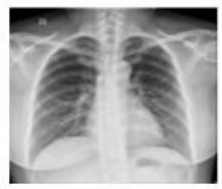

Failed normal

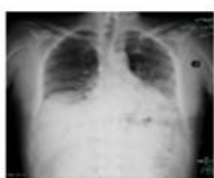

Failed covid19

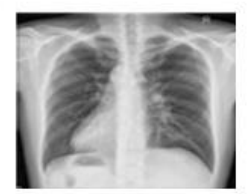

Failed normal

Figure 5. Examples of several images that failed to be detected 
Referring to Figure 5, the parameter values for the number of white patches and the percentage of white patches belong to the thorax X-ray image of the COVID-19 are below the parameter values for the number of white patches and the percentage of white patches that belong to the thorax X-ray image. This case is not by the existing hypothesis, also in the visual vision by the eye, normal thorax X-ray images have a clean condition, and there are not many white patches. This case could be due to the quality possessed by the image not included as a good quality image category. Because from a medical point of view, to get a good quality X-ray image must meet the requirements for a post anterior thorax X-ray if possible, including the following: (i) The symmetrical position for the projection of the thoracal vertebral body or spinal column of the thorax is located in the middle of the sternoclavicular joint, namely the synovial joint that connects the medial end of the clavicula to the sternum and the first rib, which has a function in helping the movement of the shoulder wrist. (ii) The X-ray condition is suitable, which is indicated by a sufficient number of rays so that the film outside the thoracic cavity is entirely black and the quality of the rays is sufficient so that the thoracic spine is visible only 3 to 4 pieces. (iii) The film covers the entire thoracic cavity, where the top of the thoracic cavity and phrenico-costal sinuses are on the right-left [37]. The success rate of segmentation testing for thorax X-ray images of COVID-19 and normal are as shown in Table 4.

Table 4. The labeling process on the segmented thorax

\begin{tabular}{|c|c|c|c|c|c|c|}
\hline No & Image & Labeling result & $\begin{array}{c}\text { Thorax area } \\
\text { (pixels) }\end{array}$ & $\begin{array}{l}\text { The number of } \\
\text { white patches }\end{array}$ & $\begin{array}{c}\text { White patches } \\
\text { area }\end{array}$ & $\begin{array}{c}\text { White patches } \\
\text { percentage }\end{array}$ \\
\hline 1. & Image_Covid1 & & 49599.1 & 44 & 28585 & 57,6321 \\
\hline 2. & Image_Covid2 & & 73076.4 & 182 & 40278,5 & 55,1183 \\
\hline 3. & Image_Covid3 & & 37973.5 & 46 & 24687.5 & 65,0124 \\
\hline 4 & Image_Normal1 & & 37709 & 27 & 17664.1 & 46,8432 \\
\hline 5. & Image_Normal2 & & 53700.1 & 57 & 23680.5 & 44,0977 \\
\hline 6 & Image_Normal3 & & 62628.1 & 53 & 30630.4 & 48,9084 \\
\hline
\end{tabular}

Referring to Table 5, the Bradley thresholding method in segmenting white patches on thorax Xrays of COVID-19 as many as 33 images with failure of 12 images, so the percentage success rate for this is $73.33 \%$. Whereas for white patches segmentation on thorax X-ray images of normal, this method succeeded in segmenting 27 images and 23 images failed, and the percentage of success was $54 \%$ for testing white patches segmentation on thorax X-rays of normal. 
Table 5. The performance of the Bradley thresholding method

\begin{tabular}{lccc}
\hline & Succeed & Failed & Performance \\
\hline COVID 19 Thorax & 33 images & 12 images & $73.33 \%$ \\
Normal Thorax & 27 images & 23 images & $54 \%$ \\
\hline
\end{tabular}

\section{CONCLUSIONS}

Segmentation using the Bradley thresholding method succeeded in obtaining the area of the thorax and white patches on the thorax X-ray image in pixels. As a result, white patches segmentation using the Bradley thresholding method can simplify the process of analyzing X-ray images for people with COVID-19 to get more accurate and precise image information. Based on the comparison of the average percentage of white patches of the two data above, there was a significant difference between thorax X-ray image data of COVID-19 patient and thorax X-ray image data of a normal patient with a difference of $5.75956 \%$ with the success rate of testing each of $73.33 \%$ and $54 \%$. For further research, we will classify the severity of the COVID-19 disease with other features that support the diagnosis of COVID19.

\section{ACKNOWLEDGEMENTS}

We would like to thank the Ministry of Education, Culture, Research, and Technology, who provided funding for this research through the "Fundamental Research" scheme with contract number 117/SP2H/LT/DRPM/2021.

\section{REFERENCES}

[1] World Health Organization, "Update on coronavirus disease in Indonesia," 2021.

[2] P. Belluck, "What Exactly Does This Virus Do to the Body," New York Times, New York, USA, 17-Mar-2020.

[3] N. N. Kinloch et al., "Evaluation of Nasopharyngeal Swab Collection Techniques for Nucleic Acid Recovery and Participant Experience: Recommendations for COVID-19 Diagnostics," Open Forum Infect. Dis., vol. 7, no. 11, pp. 1-6, 2020, doi: 10.1093/ofid/ofaa488.

[4] C. Wu et al., "Risk Factors Associated with Acute Respiratory Distress Syndrome and Death in Patients with Coronavirus Disease 2019 Pneumonia in Wuhan, China," JAMA Intern. Med., vol. 180, no. 7, pp. 934-943, 2020, doi: 10.1001/jamainternmed.2020.0994.

[5] B. Ragnesola, D. Jin, C. C. Lamb, B. H. Shaz, C. D. Hillyer, and L. L. Luchsinger, "COVID19 antibody detection using lateral flow assay tests in a cohort of convalescent plasma donors," BMC Res. Notes, vol. 13, no. 1, pp. 1-8, 2020, doi: 10.1186/s13104-020-05212-0.

[6] S. E. Gill et al., "Transcriptional profiling of leukocytes in critically ill COVID19 patients: implications for interferon response and coagulation," Intensive Care Med. Exp., vol. 8, no. 1, 2020, doi: 10.1186/s40635-020-00361-9.

[7] T. Goshen-Lago et al., "The Potential Role of Immune Alteration in the Cancer-COVID19 Equation-A Prospective Longitudinal Study," Cancers (Basel)., vol. 12, no. 2421, 2020, doi: 10.3390/cancers12092421.

[8] H. Alshazly, C. Linse, E. Barth, and T. Martinetz, "Explainable COVID-19 Detection Using Chest CT Scans and Deep Learning," Sensors, vol. 21, no. 455, 2021, doi: 10.3390/s21020455.

[9] M. Siwiak, P. Szczesny, and M. Siwiak, "From the index case to global spread: The global mobility based modelling of the COVID-19 pandemic implies higher infection rate and lower detection ratio than current estimates," PeerJ, vol. 2020, no. 7, 2020, doi: 10.7717/peerj.9548.

[10] S. Tiwari and A. Jain, "Convolutional capsule network for COVID-19 detection using radiography images," Int. J. Imaging Syst. Technol., no. February, pp. 1-16, 2021, doi: 10.1002/ima.22566.

[11] H. Cho et al., "Human endogenous retrovirus-enveloped baculoviral DNA vaccines against MERS-CoV and SARS-CoV2," npj Vaccines, vol. 6, no. 1, pp. 1-10, 2021, doi: 10.1038/s41541-021-00303-w.

[12] T. Leger et al., "Low-dose chest CT for diagnosing and assessing the extent of lung involvement of SARS-CoV-2 pneumonia using a semi quantitative score," PLoS One, vol. 15, no. 11 November, pp. 1-13, 2020, doi: 10.1371/journal.pone.0241407.

[13] L. Wang, Z. Q. Lin, and A. Wong, "COVID-Net: a tailored deep convolutional neural network design for detection of COVID-19 cases from chest X-ray images,” Sci. Rep., vol. 10, no. 1, pp. 1-13, 2020, doi: 10.1038/s41598-020-76550-Z.

[14] A. Jaffe, M. L. Schilsky, R. Deshpande, and R. Batra, "Liver Transplantation in the Time of COVID19: Barriers and Ethical Considerations for Management and Next Steps," Hepatol. Commun., vol. 4, no. 9, pp. 1242-1256, 2020, doi: 10.1002/hep4.1568.

[15] D. A. O'Connell et al., "Recommendations from the Canadian Association of Head and Neck Surgical Oncology for the Management of Head and Neck Cancers during the COVID-19 pandemic," J. Otolaryngol. - Head Neck Surg., vol. 49, no. 1, pp. 1-8, 2020, doi: 10.1186/s40463-020-00448-z.

[16] A. E. Dhamad and M. A. Abdal Rhida, "COVID-19: Molecular and serological detection methods," PeerJ, vol. 8, pp. 1-19, 2020, doi: 10.7717/peerj.10180.

[17] F. Keihanian and L. Bigdelu, "Cardiovascular considerations in COVID-19: A comprehensive review," Ther. Clin. Risk Manag., vol. 16, pp. 1089-1097, 2020, doi: 10.2147/TCRM.S264377. 
[18] The COVID-19 APHP-Universities-INRIA-INSERM Group, "Early indicators of intensive care unit bed requirement during the COVID-19 epidemic: A retrospective study in Ile-de-France region, France," PLoS One, vol. 15, no. November 11, pp. 1-13, 2020, doi: 10.1371/journal.pone.0241406.

[19] R. Supriyanti, A. K. Marchel, Y. Ramadhani, and H. B. Widodo, "Coronal slice segmentation using a watershed method for early identification of people with Alzheimer's," TELKOMNIKA (Telecommunication Comput. Electron. Control., vol. 19, no. 1, pp. 63-72, 2020, doi: 10.12928/TELKOMNIKA.V19I1.15142.

[20] R. Supriyanti, A. R. Subhi, E. J. Ashari, F. Ahmad, Y. Ramadhani, and H. B. Widodo, "Simple Classification of the Alzheimer's Severity in Supporting Strengthening the Diagnosis of Patients based on ROC Diagram," IOP Conf. Ser. Mater. Sci. Eng., vol. 982, no. 1, 2020, doi: 10.1088/1757-899X/982/1/012007.

[21] R. Supriyanti, A. Rifai, Y. Ramadhani, and W. Siswandari, "Influence of camera types in histogram distribution on morphological identification of myeloblast cell based image segmentation," J. Phys. Conf. Ser., vol. 1321, no. 3, 2019, doi: 10.1088/1742-6596/1321/3/032017.

[22] R. Supriyanti, A. K. Marchel, Y. Ramadhani, and H. B. Widodo, "Coronal slice segmentation using a watershed method for early identification of people with Alzheimer's," TELKOMNIKA (Telecommunication Comput. Electron. Control., vol. 19, no. 1, pp. 63-72, 2020, doi: 10.12928/TELKOMNIKA.V19I1.15142.

[23] R. Supriyanti, A. Haeromi, Y. Ramadhani, and W. Siswandari, "Unsupervised Method for Calculating Diameter and Number of Leukocyte Cells," IOP Conf. Ser. Mater. Sci. Eng., vol. 982, no. 1, 2020, doi: 10.1088/1757899X/982/1/012006.

[24] T. Ercleve, "Normal Chest X-Ray," Life In The Fastlane, 2020. [Online]. Available: https://litfl.com/normal-chestX-ray/. [Accessed: 25-Mar-2020].

[25] L. Noviasari and Mashoedah, "Classification of Thorax X-ray Results on Corona Virus Infection Based on Internet of Things (IoT),” J. Phys. Conf. Ser., vol. 1737, no. 1, 2021, doi: 10.1088/1742-6596/1737/1/012001.

[26] J. Ferguson, K. Blyth, I. McInnes, A. Biankin, and R. Jones, "P258 ASTERIX: Adaptive stratification of COVID19 to facilitate endotype-directed intervention studies," Thorax, vol. 76, no. 1, 2020, doi: 10.1136/thorax-2020-BTSabstracts.402.

[27] M. Soltan, L. Crowley, C. R. Melville, J. Varney, and S. Cassidy, "L12 To what extent are social determinants of health, including household overcrowding, air pollution and housing quality deprivation, modulators of presentation, ITU admission and outcomes among patients with SARS-COV-2 infection in an urban catchment ar," Thorax, vol. 76, no. 1, 2020, doi: 10.1136/thorax-2020-BTSabstracts.414.

[28] M. A. Man, R.-M. Rajnoveanu, N. S. Motoc, C. I. Bondor, and A. F. Chis, "Neutrophil-to-lymphocyte ratio, platelets-to-lymphocyte ratio, and eosinophils correlation with high-resolution computer tomography severity score in COVID-19 patients," PLoS One, vol. 16, no. 6, 2021, doi: 10.1371/journal.pone.0252599.

[29] N. Mayer, P. Perikleous, G. Doukas, K. De Rome, and H. Bruijnen, "P31 Thoracic surgery in the COVID-19 era: a tertiary single centre report," Thorax, vol. 76, no. 1, 2020, doi: 10.1136/thorax-2020-BTSabstracts.176.

[30] M. Manzo and S. Pellino, "Fighting Together against the Pandemic: Learning Multiple Models on Tomography Images for COVID-19 Diagnosis," AI, vol. 2, no. 2, 2021, doi: 10.3390/ai2020016.

[31] F. G. Kavanagh, D. Callanan, C. Connolly, D. Brinkman, and C. Fitzgerald, "Pre-operative testing for SARS-CoV-2 and outcomes in otolaryngology surgery during the pandemic: A multi-center experience," Laryngoscope Investig. Otolaryngol., vol. 6, no. 4, 2021, doi: 10.1002/lio2.613.

[32] M. Servi, A. Zulli, Y. Volpe, R. Furferi, and L. Puggelli, "Handheld Optical System for Pectus Excavatum Assessment," Appl. Sci., vol. 11, no. 4, 2021, doi: 10.3390/app11041726.

[33] A. H. Osman;, H. M. Aljahdali, S. M. Altarrazi; and A. Ahmed, "SOM-LWL method for identification of COVID19 on chest X-rays," PLoS One, vol. 16, no. 2, 2021, doi: 10.1371/journal.pone.0247176.

[34] P. Correale, M. Caracciolo, M. Bilotta, Federico; Conte, and M. Cuzzola, "Therapeutic effects of adenosine in high flow $21 \%$ oxygen aereosol in patients with Covid19-pneumonia," PLoS One, vol. 15, no. 10, 2020, doi: 10.1371/journal.pone.0239692.

[35] A. Holborow, H. Asad, L. Porter, P. Tidswell, and C. Johnston, "The clinical sensitivity of a single SARS-CoV-2 upper respiratory tract RT-PCR test for diagnosing COVID-19 using convalescent antibody as a comparator," Clin. Med. (Northfield. Il)., vol. 20, no. 6, 2020, doi: 10.7861/clinmed.2020-0555.

[36] D. Bradley and G. Roth, "Adaptive Thresholding using the Integral Image," J. Graph. Tools, vol. 12, no. 2, 2007, doi: 10.1080/2151237x.2007.10129236.

[37] S. Asriyani, "Radiological Methods of Respiratory Investigation," in Basic of Chest X Ray, Makassar, 2017.

\section{BIOGRAPHIES OF AUTHORS}

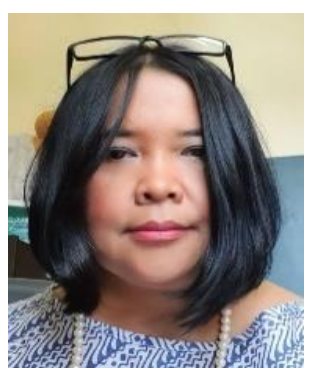

Retno Supriyanti is a professor at Electrical Engineering Department, Jenderal Soedirman University, Indonesia. She received her PhD in March 2010 from Nara Institute of Science and Technology Japan. Also, she received her M.S degree and Bachelor degree in 2001 and 1998, respectively, from Electrical Engineering Department, Gadjah Mada University Indonesia. Her research interests include image processing, computer vision, pattern recognition, biomedical application, e-health, tele-health and telemedicine. 


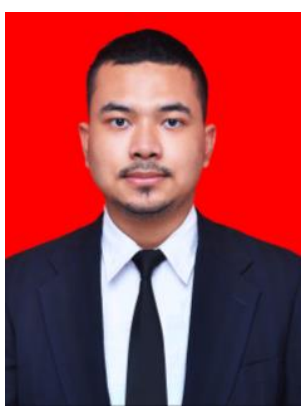

Muhammad Alqaaf received his Bachelor degree from Electrical Engineering Depratment, Jenderal Soedirman University Indonesia. His research interest Image Processing field

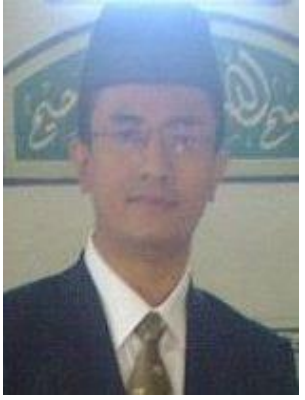

Yogi Ramadhani is an academic staff at Electrical Engineering Department, Jenderal Soedirman University, Indonesia. He received his MS Gadjah Mada Universirt Indonesia, and his Bachelor degree from Jenderal Soedirman University Indonesia. His research interest including Computer Network, Decision Support Syetem, Telemedicine and Medical imaging

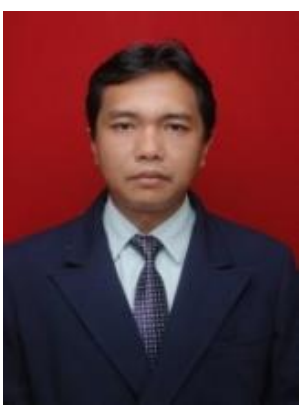

Haris Budi Widodo is is an academic staff at Public Health Department, Jenderal Soedirman University, Indonesia. He received his Ph.D from AirlanggaUniversity Indonesia. Also, He received his M.S degree and bachelor degree from Gadjah Mada University Indonesia. His research interest including public health, e-health and telemedicine 